\title{
Model-independent determinations of the electron EDM and the role of diamagnetic atoms
}

\author{
Timo Fleig ${ }^{a}$ and Martin Jung ${ }^{b}$ \\ ${ }^{a}$ Laboratoire de Chimie et Physique Quantiques, IRSAMC, \\ Université Paul Sabatier Toulouse III, \\ 118 Route de Narbonne, F-31062 Toulouse, France \\ ${ }^{b}$ Excellence Cluster Universe, Technische Universität München, \\ Boltzmannstr. 2, D-85748 Garching, Germany \\ E-mail: timo.fleig@irsamc.ups-tlse.fr, martin.jung@tum.de
}

ABSTRACT: We perform model-independent analyses extracting limits for the electric dipole moment of the electron and the P,T-odd scalar-pseudoscalar (S-PS) nucleon-electron coupling from the most recent measurements with atoms and molecules. The analysis using paramagnetic systems, only, is improved substantially by the inclusion of the recent measurement on $\mathrm{HfF}^{+}$ions, but complicated by the fact that the corresponding constraints are largely aligned, owing to a general relation between the coefficients for the two contributions. Since this same relation does not hold in diamagnetic systems, it is possible to find atoms that provide essentially orthogonal constraints to those from paramagnetic ones. However, the coefficients are suppressed in closed-shell systems and enhancements of $\mathrm{P}, \mathrm{T}$-odd effects are only prevalent in the presence of hyperfine interactions. We formulate the hyperfine-induced time-reversal-symmetry breaking S-PS nucleon-electron interaction in general atoms in a mixed perturbative and variational approach, based on electronic Dirac-wavefunctions including the effects of electron correlations. The method is applied to the $\mathrm{Hg}$ atom, yielding the first direct calculation of the coefficient of the S-PS nucleonelectron coupling in a diamagnetic system. This results in additionally improved modelindependent limits for both the electron EDM and the nucleon-electron coupling from the global fit. Finally we employ this fit to provide indirect limits for several paramagnetic systems under investigation.

Keywords: Beyond Standard Model, CP violation

ARXiv EPRINT: 1802.02171 


\section{Contents}

1 Introduction 1

2 Theoretical framework $\quad 2$

3 S-PS-ne coefficient in atomic mercury 5

$\begin{array}{llr}4 & \text { Phenomenological consequences } & 8\end{array}$

5 Conclusions and outlook 12

\section{Introduction}

Electric dipole moments (EDMs) provide a competitive means to search for new physics (NP), complementary to strategies like direct searches at hadron colliders, but also to other indirect searches, for instance using flavour-changing processes. Searches for new sources of CP violation are strongly motivated by the fact that the Standard Model (SM) is not capable of explaining the baryon asymmetry of the universe quantitatively [1-3]. The exceptional sensitivity of EDM searches is due to the combination of experimental precision with a very specific Standard Model (SM) background: it is tiny for paramagnetic systems and due to potential strong $C P$ violation in other systems. The latter is generically large, i.e. naively orders of magnitude above present limits, but has not been observed so far. Experimental tests for EDMs involve typically rather complex systems like atoms or molecules. The discovery of a finite EDM in any of these systems would be a major discovery, independent of its source being NP or strong CP violation. However, reliably interpreting these measurements in terms of fundamental parameters of a given NP model requires precise knowledge of their relations. These are established proceeding via a series of effective field theories, rendering a large part of the analysis model- and system-independent, see e.g. refs. [4-12] for recent reviews. The corresponding complex matrix elements on the atomic, nuclear and QCD levels often involve large uncertainties, which sometimes prohibit to fully exploit the experimental information, see refs. $[9,13]$ for recent detailed discussions.

This article presents a new method for the rigorous calculation of the coefficient of the scalar-pseudoscalar nucleon-electron (S-PS-ne) interaction in diamagnetic systems. For this contribution so far only rough estimates exist, due to the fact that it vanishes to leading order in the electromagnetic interaction, even in the presence of an external electric field. In this paper we consider Mercury $(\mathrm{Hg})$ which provides the strongest experimental limit on an EDM so far [14]. The determination of this coefficient provides a competitive limit on the (NP-induced) strength of the corresponding interaction. It is also of special interest for 
the model-independent extraction of the electron EDM: in principle, paramagnetic systems can be used to obtain both coefficients, taking into account potential cancellations $[15,16]$; however, a problem arises from the fact that all paramagnetic systems constrain a similar combination of these two contributions [15]. Diamagnetic systems generally give independent constraints, thereby improving the model-independent extraction of both coefficients significantly [16]. Our results can therefore be used to constrain different classes of NP models, requiring less restrictive assumptions.

This article proceeds as follows: in the following section we present a method for the direct calculation of S-PS-ne enhancements in closed-shell atoms and molecules. Section 3 describes its application to the $\mathrm{Hg}$ atom, and in section 4 we investigate the phenomenological consequences of the present study. In the final section we conclude and discuss the implications of our findings for future work.

\section{Theoretical framework}

The calculation of the dominant contribution induced by the S-PS-ne interaction in diamagnetic systems requires the inclusion of the hyperfine interaction on top of the corresponding calculation in paramagnetic systems, since its expectation value vanishes to leading order in a closed-shell atom, due to a vanishing spin density near its nucleus [17, 18]. The nuclear current at the origin, corresponding to the magnetic moment of the nucleus, polarizes the closed atomic shells and leads to non-zero values. In a traditional setup this would require a three-fold expansion in the S-PS-ne interaction, the external electric field and the hyperfine interaction. Instead, we here start from a 0th-order electronic-structure problem

$$
\hat{H}^{(0)}\left|\psi_{K}^{(0)}\right\rangle=\varepsilon_{K}^{(0)}\left|\psi_{K}^{(0)}\right\rangle
$$

where $H^{(0)}$ is the atomic Dirac-Coulomb Hamiltonian including the perturbation due to a homogeneous external electric field $\mathbf{E}_{\text {ext }}$, with the nucleus placed at the origin:

$$
\begin{aligned}
\hat{H}^{(0)} & :=\hat{H}^{\text {Dirac-Coulomb }}+\hat{H}^{\text {Int-Dipole }} \\
& =\sum_{j}^{N}\left[c \boldsymbol{\alpha}_{j} \cdot \mathbf{p}_{j}+\beta_{j} c^{2}+\frac{Z}{r_{j}} \mathbb{1}_{4}\right]+\sum_{j, k>j}^{N} \frac{1}{r_{j k}} \mathbb{1}_{4}+\sum_{j} \mathbf{r}_{j} \cdot \mathbf{E}_{\text {ext }} \mathbb{1}_{4},
\end{aligned}
$$

where the indices $j, k$ run over $N$ electrons, $Z$ is the proton number $(N=Z$ for neutral atoms), and $\boldsymbol{\alpha}, \beta$ are standard Dirac matrices. We use atomic units (a.u.) throughout ( $e=m_{0}=\hbar=1$ ). Since we solve eq. (2.1) variationally (i.e. by diagonalization), the effect of the external electric field in $\left|\psi_{K}^{(0)}\right\rangle$ is taken into account to all orders in perturbation theory. These states are technically electronic configuration interaction (CI) vectors [19].

The first-order perturbed wavefunction due to the magnetic hyperfine interaction can be written as

$$
\left|\psi_{J}^{(1)}\right\rangle=\left|\psi_{J}^{(0)}\right\rangle+\sum_{K \neq J} \frac{\left\langle\psi_{K}^{(0)}\left|\hat{H}_{\mathrm{HF}}^{(1)}\right| \psi_{J}^{(0)}\right\rangle}{\varepsilon_{J}^{(0)}-\varepsilon_{K}^{(0)}}\left|\psi_{K}^{(0)}\right\rangle,
$$


where in practice the summation is carried out over a restricted set of CI vectors. The perturbation sum in eq. (2.3) will only be well-defined if $\left|\psi_{J}^{(0)}\right\rangle$ is a non-degenerate state, which is the case for the electronic ground state of a closed-shell atom.

Since $\hat{H}_{\mathrm{HF}}^{(1)}$ is a totally symmetric operator with respect to all valid symmetry operations of the system including the external field (axial symmetry), the sum in eq. (2.3) includes only states of the same irreducible representation as the reference state $\left|\psi_{J}^{(0)}\right\rangle$. The magnetic hyperfine Hamiltonian reads

$$
\hat{H}_{\mathrm{HF}}=-\frac{1}{2 c m_{p}} \frac{\mu \boldsymbol{I}}{I} \cdot \sum_{i=1}^{n} \frac{\boldsymbol{\alpha}_{i} \times \boldsymbol{r}_{i}}{r_{i}^{3}},
$$

where $\mu=g I$ is the nuclear magnetic moment, $g$ the nuclear $g$-factor, $m_{p}$ the proton mass and $\boldsymbol{I}$ the nuclear spin. The minus sign in eq. (2.4) relates to the charge of an electron in a.u. The hyperfine Hamiltonian can also be written as $\hat{H}_{\mathrm{HF}}^{(1)}=\boldsymbol{I} \mathcal{A} \boldsymbol{J}$, where $\mathcal{A}$ is the rank 2 cartesian hyperfine interaction tensor and $\boldsymbol{J}$ is the total electronic angular momentum. It is, therefore, generally a sum of nine terms that due to $\mu:=\left\langle I, M_{I}=I\left|\hat{\mu}_{z}\right| I, M_{I}=I\right\rangle$ and $\boldsymbol{\mu} \propto \boldsymbol{I}$ reduces to $\hat{H}_{\mathrm{HF}}^{(1)}=I_{z}\left(\mathcal{A}_{z x} J_{x}+\mathcal{A}_{z y} J_{y}+\mathcal{A}_{z z} J_{z}\right)$. The required matrix elements are defined as follows:

$$
\left(A_{z k}\right)_{M N}=-\frac{\mu\left[\mu_{N}\right]}{2 c I m_{p}} \sum_{i=1}^{n}\left\langle\psi_{M}^{(0)}\left|\left(\frac{\boldsymbol{\alpha}_{i} \times \boldsymbol{r}_{i}}{r_{i}^{3}}\right)_{k}\right| \psi_{N}^{(0)}\right\rangle
$$

where $k$ is a cartesian component and the nuclear magnetic moment enters in units of the nuclear magneton $\mu_{N}=\frac{1}{2 c m_{p}}$ (in a.u.).

For evaluating the S-PS-ne coefficient in the atom we use the effective Hamiltonian operator $[20]$

$$
\hat{H}_{\text {S-PS-ne }}(S)=\imath \frac{G_{F}}{\sqrt{2}} A C_{S} \sum_{e} \gamma_{e}^{0} \gamma_{e}^{5} \rho\left(\mathbf{r}_{e}\right)
$$

where $G_{F}$ is the Fermi constant, $A$ the nucleon number, $C_{S}$ the dimensionless S-PS-ne coupling constant, $\rho$ the normalized nuclear charge density, and $\gamma^{\mu}$ are standard Dirac matrices. Given the smallness of this interaction, even compared to the hyperfine interaction, higher-order perturbative corrections are clearly negligible. Given, furthermore, the CP-conserving nature of the hyperfine interaction, the energy shift of a given atomic state indicating $\mathrm{CP}$ violation can to leading order be written as

$$
(\Delta \varepsilon)_{J}=\frac{1}{\left\langle\psi_{J}^{(1)} \mid \psi_{J}^{(1)}\right\rangle}\left\langle\hat{H}_{\text {S-PS-ne }}\right\rangle_{\psi_{J}^{(1)}}
$$

The atomic EDM in terms of the S-PS-ne interaction is a function of the polarizing external electric field $E_{\text {ext }}$, and so

$$
d_{a}=-\lim _{E_{\text {ext }} \rightarrow 0}\left[\frac{\partial(\Delta \varepsilon)}{\partial E_{\text {ext }}}\right] \approx-A C_{S} \frac{G_{F}}{\sqrt{2}} \frac{\left\langle\imath \sum_{e} \gamma_{e}^{0} \gamma_{e}^{5} \rho\left(\mathbf{r}_{e}\right)\right\rangle_{\psi^{(1)}\left(E_{\text {ext }}\right)}}{E_{\text {ext }}\left\langle\psi^{(1)} \mid \psi^{(1)}\right\rangle} \equiv \alpha_{C_{S}} C_{S},
$$

where the approximation holds in the linear regime which is assured by external fields chosen significantly smaller than the internal ones and we have introduced $\alpha_{C_{S}}$, the atomic 
S-PS-ne coefficient factor. In the present case $E_{\text {ext }}(\mathrm{Hg})=0.00024$ a.u. This leads to shifts of the energies $\varepsilon_{K}^{(0)}$ (see eq. (2.1)) on the order of $10^{-6}$ a.u. for Hg. CI vectors are consequently optimized such that the energies $\varepsilon_{K}^{(0)}$ are converged to at least $10^{-9}$ a.u.

We now focus on the evaluation of the normalized expectation value, part of the expression on the right-hand side of eq. (2.8),

$$
\begin{aligned}
& \frac{1}{\left\langle\psi_{J}^{(1)} \mid \psi_{J}^{(1)}\right\rangle}\left\langle\psi_{J}^{(1)}\left|\imath \sum_{e} \gamma_{e}^{0} \gamma_{e}^{5} \rho\left(\mathbf{r}_{e}\right)\right| \psi_{J}^{(1)}\right\rangle= \\
& \frac{1}{\left\langle\psi_{J}^{(1)} \mid \psi_{J}^{(1)}\right\rangle}\left[\sum_{K \neq J} \frac{\left\langle\psi_{K}^{(0)}\left|\hat{H}_{\mathrm{HF}}^{(1)}\right| \psi_{J}^{(0)}\right\rangle}{\varepsilon_{J}^{(0)}-\varepsilon_{K}^{(0)}}\left\langle\psi_{J}^{(0)}\left|\imath \sum_{e} \gamma_{e}^{0} \gamma_{e}^{5} \rho\left(\mathbf{r}_{e}\right)\right| \psi_{K}^{(0)}\right\rangle\right. \\
& \\
&\left.+\sum_{K \neq J} \frac{\left\langle\psi_{J}^{(0)}\left|\hat{H}_{\mathrm{HF}}^{(1)}\right| \psi_{K}^{(0)}\right\rangle}{\varepsilon_{J}^{(0)}-\varepsilon_{K}^{(0)}}\left\langle\psi_{K}^{(0)}\left|\imath \sum_{e} \gamma_{e}^{0} \gamma_{e}^{5} \rho\left(\mathbf{r}_{e}\right)\right| \psi_{J}^{(0)}\right\rangle\right],
\end{aligned}
$$

up to higher-order terms in the hyperfine interaction, where we used the hyperfineperturbed wavefunction from eq. (2.3). The leading term in this equation (for open-shell atoms) vanishes for closed-shell atoms, and is omitted. This conclusion has also been tested numerically in the present work. Transition matrix elements of the type $\left\langle\psi_{K}^{(0)}\left|\hat{H}_{\mathrm{HF}}^{(1)}\right| \psi_{J}^{(0)}\right\rangle$ and $\left\langle\psi_{K}^{(0)}\left|\imath \sum_{e} \gamma_{e}^{0} \gamma_{e}^{5} \rho\left(\mathbf{r}_{e}\right)\right| \psi_{J}^{(0)}\right\rangle$, required for evaluating these two terms, can be readily made available using the developed methodology in refs. [21, 22]. The practical problem is then to provide a sufficient set of CI states for the perturbation sum. The final expression for evaluating the S-PS-ne coefficient is, therefore,

$$
\alpha_{C_{S}}\left(\psi_{J}\right)=\frac{-A \frac{G_{F}}{\sqrt{2}}}{E_{\text {ext }}\left\langle\psi_{J}^{(1)} \mid \psi_{J}^{(1)}\right\rangle}\left[\sum_{K \neq J} \frac{\left\langle\psi_{K}^{(0)}\left|\hat{H}_{\mathrm{HF}}^{(1)}\right| \psi_{J}^{(0)}\right\rangle}{\varepsilon_{J}^{(0)}-\varepsilon_{K}^{(0)}}\left\langle\psi_{J}^{(0)}\left|\imath \sum_{e} \gamma_{e}^{0} \gamma_{e}^{5} \rho\left(\mathbf{r}_{e}\right)\right| \psi_{K}^{(0)}\right\rangle+\text { h.c. }\right]
$$

For convenience, we use in the following also the S-PS-ne ratio $S$ (in analogy to the electron EDM enhancement $R$ and not to be confused with the nuclear Schiff moment, also denoted $S$ in the literature), defined as

$$
S:=\frac{d_{a}}{A C_{S} \frac{G_{F}}{\sqrt{2}}}=\frac{\alpha_{C_{S}}}{A \frac{G_{F}}{\sqrt{2}}} \approx-\frac{\left\langle\imath \sum_{e} \gamma_{e}^{0} \gamma_{e}^{5} \rho\left(\mathbf{r}_{e}\right)\right\rangle_{\psi^{(1)}\left(E_{\mathrm{ext}}\right)}}{E_{\mathrm{ext}}\left\langle\psi^{(1)} \mid \psi^{(1)}\right\rangle}
$$

In order to facilitate comparison with the literature, we note that the states $\left|\psi_{K}^{(0)}\right\rangle$ can be considered as wavefunctions perturbed to infinite order by $\mathbf{E}$, and so the expression in eq. (2.9) contains terms of third order of the type

$$
\sum_{K, N \neq J} \frac{\left\langle\psi_{J}^{(0)}\left|\sum_{i} \hat{r}_{z}(i)\right| \psi_{N}^{(0)}\right\rangle E_{z}\left\langle\psi_{K}^{(0)}\left|\hat{H}_{\mathrm{HF}}^{(1)}\right| \psi_{J}^{(0)}\right\rangle}{\left(\varepsilon_{J}^{(0)}-\varepsilon_{N}^{(0)}\right)\left(\varepsilon_{J}^{(0)}-\varepsilon_{K}^{(0)}\right)}\left\langle\psi_{N}^{(0)}\left|\imath \sum_{e} \gamma_{e}^{0} \gamma_{e}^{5} \rho\left(\mathbf{r}_{e}\right)\right| \psi_{K}^{(0)}\right\rangle,
$$


plus higher-order contributions in $\mathbf{E}$, where $\left|\psi_{N}^{(0)}\right\rangle$ is now an unperturbed eigenstate of the plain atomic Dirac-Coulomb Hamiltonian without external electric field. The terms in eq. (2.12) are just the equivalent of the electron EDM contribution via magnetic hyperfine interaction to an atomic EDM, as given by Flambaum and Khriplovich in reference [20], eq. (17). These third-order terms, declared important but left untreated in reference [23], are taken into account in the present approach. Moreover, the higher-order contributions in $\mathbf{E}$ are included automatically in the present approach.

\section{S-PS-ne coefficient in atomic mercury}

For our zeroth-order atomic wavefunctions the quantum number $M_{J}$, corresponding to the projection of the total angular momentum onto the axis defined by the external electric field, is an exact quantum number and characterizes an irreducible representation of the axial double point group. Since the external perturbation is small, the quantum number $J$ is still approximately valid and we denote CI states in the approximate Russell-Saunders picture as ${ }^{M} L_{J, M_{J}}$, where $M$ is the spin multiplicity. The S-PS-ne interaction Hamiltonian in eq. (2.6) is rotationally invariant; as a consequence, $\left\langle M_{J}\left|\hat{H}_{\mathrm{S}-\mathrm{PS}-\mathrm{ne}}\right| M_{J}^{\prime}\right\rangle=0$ for $M_{J} \neq M_{J}^{\prime}$, which reduces the perturbation sum in eq. (2.9) to states from the irreducible representation $M_{J}=0$, a computational advantage which we exploit.

Applying the framework developed in the last section to Mercury, a consistent finding in all our calculations is that among the 35 energetically lowest-lying excited states of symmetry $M_{J}=0$ only three states contribute sizably to the perturbation sum eq. (2.9) determining $\alpha_{C_{S}}$, namely $\psi_{K}^{(0)} \in\left\{{ }^{3} P_{0, M_{J}=0}\left(5 d^{10} 6 s 6 p\right),{ }^{3} S_{1, M_{J}=0}\left(5 d^{10} 6 s 7 s\right),{ }^{3} P_{0, M_{J}=0}\left(5 d^{10} 6 s 7 p\right)\right\}$. This finding can be understood qualitatively analyzing the product of matrix elements in eq. (2.9): for contributions of the type

$$
\left\langle{ }^{3} P_{0, M_{J}=0}\left|\hat{H}_{\mathrm{HF}}^{(1)}\right|{ }^{1} S_{0, M_{J}=0}\right\rangle\left\langle{ }^{1} S_{0, M_{J}=0}\left|\imath \sum_{e} \gamma_{e}^{0} \gamma_{e}^{5} \rho\left(\mathbf{r}_{e}\right)\right|{ }^{3} P_{0, M_{J}=0}\right\rangle
$$

the off-diagonal S-PS-ne matrix element is large due to the parity-odd excitation $6 s \rightarrow n p$ characterizing the excited state, and the off-diagonal hyperfine matrix element is nonnegligible due to $s p$-mixing via the external electric field. For the other leading type of contribution,

$$
\left\langle{ }^{3} S_{1, M_{J}=0}\left|\hat{H}_{\mathrm{HF}}^{(1)}\right|{ }^{1} S_{0, M_{J}=0}\right\rangle\left\langle{ }^{1} S_{0, M_{J}=0}\left|\imath \sum_{e} \gamma_{e}^{0} \gamma_{e}^{5} \rho\left(\mathbf{r}_{e}\right)\right|{ }^{3} S_{1, M_{J}=0}\right\rangle,
$$

the off-diagonal S-PS-ne matrix element is now two orders of magnitude smaller than in the above case - for obvious reasons related to symmetry - , but the off-diagonal hyperfine matrix element becomes almost three orders of magnitude larger than for the previous mechanism. This is explained by the fact that the excited state ${ }^{3} S_{1}$ exhibits a non-vanishing spin-density near the nucleus.

Results from many-body models of different sophistication are compiled in table 1 . The S-PS-ne coefficient is largely converged when at least the 12 lowest-lying $M_{J}=0$ states are included in the perturbation sum, since then the three main contributors are covered. 


\begin{tabular}{|llccc|}
\hline Basis/cutoff & \# of CI states $M_{J}=0 /$ Model/X & $\frac{\text { Meandev. }}{\%}$ & $\frac{S}{10^{-2} a . u .}$ & $\frac{\alpha_{C_{S}}}{10^{-22} e \mathrm{~cm}}$ \\
\hline DZ/150 a.u. & 4/M12/6p7s7p6d5f8p8s7d & & -3.3 & -5.4 \\
DZ/150 a.u. & 16/M12/6p7s7p6d5f8p8s7d & & -2.3 & -3.8 \\
TZ/50 a.u. & 12/M12/6p7s7p & 6.1 & -2.1 & -3.5 \\
TZ/50 a.u. & 12/M20/6p7s7p & & -2.1 & -3.5 \\
TZ/50 a.u. & 12/M12/6p7s7p6d8p8s & 5.4 & -2.2 & -3.7 \\
TZ/50 a.u. & 29/M12/6p7s7p6d8p8s9p9s10p10s & 6.2 & -2.22 & -3.67 \\
\hline
\end{tabular}

Table 1. S-PS-ne interaction ratio $S$ for the ${ }^{1} S_{0}$ ground state of the ${ }^{199} \mathrm{Hg}$ isotope, $I=1 / 2$, $\mu\left({ }^{199} \mathrm{Hg}\right)=+0.5058855$ [24], $E_{\mathrm{Ext}}=0.00024$ a.u.; CI models M12: 12 electrons correlated, Single, Double and Triple excitations from occupied space into X, Single and Double excitations into the remaining virtual space (SDT12-X-SD12); M20: S8-SDT12-X-SD20. DZ and TZ denote Dyall's Gaussian atomic basis sets [25, 26] including 1f,1g valence- and core-correlating exponents (DZ) and $2 \mathrm{f}, 4 \mathrm{~g}, 1 \mathrm{~h}$ valence- and core-correlating and valence-polarizing exponents (TZ), resulting in a total of $24 \mathrm{~s}, 19 \mathrm{p}, 12 \mathrm{~d}, 8 \mathrm{f}, 1 \mathrm{~g}$ for DZ and 30s,24p,15d,11f,4g,1h functions for TZ. The mean deviation concerns the difference of the calculated excited-state energies from experiment [27]. The Hg nucleus is described by a Gaussian charge distribution [28] with exponent $\zeta=1.4011788914 \times 10^{8}$.

It is furthermore important that the extent of the active spinor space is sufficient, as can be seen from the results for different values of $X$, the parameter defining the atomic functions constituting the space into which triple excitations are allowed. The remaining virtual spinors up to the cutoff threshold are allowed to be up to doubly occupied, in order to include dynamic electron correlation effects for all states described to lowest order by the structure of the active space. Correlation effects between $5 s, 5 p$ and valence electrons are tested through the model including 20 electrons and are seen to be small.

For the purpose of estimating the contribution from higher-lying excited states we use a larger basis set, denoted QZ and consisting of 34s,30p,19d,13f,4g,2h functions. Due to computational demand the model M12 is limited to X-SDT12 with X set to the value $7 \mathrm{p} 7 \mathrm{~s} 8 \mathrm{p} 9 \mathrm{p} 8 \mathrm{~s} 10 \mathrm{p} 9 \mathrm{~s}$ with reference to table 1 . This means that correlation effects are largely neglected for a large set of small contributions, $\approx 100$ states with $M_{J}=0$. We observe that only two notable contributions occur, and only in the energetically lower half, indicating that the contributions as expected fall off as energy and principal quantum number of the involved states increase. With the resulting enhancement correction $\Delta S(\mathrm{QZ})$, where $S$ is defined in eq. (2.11), our final value is obtained as follows:

$$
S(\mathrm{TZ})+\Delta S(\mathrm{QZ})=(-2.22+0.53) \times 10^{-2} \text { a.u. }=-1.69 \times 10^{-2} \text { a.u. }
$$

The uncertainty of this value is estimated by linearly adding the errors from the energy denominator $(6.2 \%$, "mean deviation" in table 1$)$, and uncertainties from atomic basis set (3.5\%), outer-core correlations $(1.5 \%)$, and higher excitation ranks $(5 \%$, estimated from comparable previous calculations of S-PS-ne enhancements, see refs. [29, 30]). To this uncertainty of $16 \%$ on the base value $S(\mathrm{TZ})$ we add an uncertainty of $30 \%$ times the relative weight $(0.24)$ of the correction $\Delta S(\mathrm{QZ})$, i.e. $7.2 \%$, resulting in a total uncertainty of $23 \%$ for $\alpha_{C_{S}}$, which we consider very conservative. Note that adding the individual terms 


\begin{tabular}{|lccc|}
\hline Method & Ref. & $C_{C_{T}}$ & $\alpha_{C_{S}} /\left(10^{-22} e \mathrm{~cm}\right)$ \\
\hline RPA & {$[32]$} & -6.0 & $(-6.0)$ \\
MCDHF & {$[33]$} & -4.8 & $(-4.8)$ \\
CI+MBPT & {$[23]$} & -5.1 & $(-5.1)$ \\
PRCC & {$[34]$} & -4.3 & $(-4.3)$ \\
$\operatorname{CCSD}^{(\infty)}$ & {$[12]$} & -3.4 & $(-3.4)$ \\
$\operatorname{CCSD}_{\mathrm{p}} \mathrm{T}(+)$ & {$[35]$} & -4.0 & $(-4.0)$ \\
$\mathrm{CCSD}_{\mathrm{p}} \mathrm{T}(+)$ & {$[36]$} & -3.2 & $(-3.2)$ \\
$\mathrm{NCCSD}$ & {$[37]$} & -3.3 & $(-3.3)$ \\
\hline Chupp et al. (est.) & {$[11,38]$} & & $(-5.9)$ \\
Engel et al. (est.) & {$[9]$} & & $(-8.1)$ \\
\hline This work & & $(-2.8)$ & $\mathbf{- 2 . 8}$ \\
\hline
\end{tabular}

Table 2. Comparison of the direct calculation presented here with previous calculations of $\alpha_{C_{S}}$, using calculations of $\alpha_{C_{T}}$ and the phenomenological relation eq. (3.3) (indicated by parentheses around the result, $\left.\mu_{H g}=0.506\right)$. The literature values are ordered as to increasing sophistication of the treatment of dynamic electron correlation. Numerically the conversion factor for Mercury reads $\alpha_{C_{S}}^{\mathrm{Hg}}=10^{-2} \alpha_{C_{T}}^{\mathrm{Hg}} /(\langle\boldsymbol{\sigma}\rangle \cdot \mathbf{I} / I)$, and a simple shell model for the nucleus is used, yielding $\langle\boldsymbol{\sigma}\rangle \cdot \mathbf{I} / I=-1 / 3$.

in quadrature, as commonly done in the literature, would result in an uncertainty of $11 \%$. From these considerations, we finally obtain from eq. (3.1) the S-PS-ne interaction constant

$$
\alpha_{C_{S}}=-2.8(6) \times 10^{-22} e \mathrm{~cm} .
$$

An indirect determination of $\alpha_{C_{S}}$ is obtained via the coefficient of the P,T-odd tensor interaction, using the phenomenological relation $[18,20,31]$

$$
\frac{\langle\boldsymbol{\sigma}\rangle \cdot \mathbf{I}}{I} \alpha_{C_{S}}=5.3 \times 10^{-4}\left(1+0.3 Z^{2} \alpha^{2}\right) A^{2 / 3} \mu_{A} \alpha_{C_{T}},
$$

where $\langle\boldsymbol{\sigma}\rangle C_{T} \equiv\left\langle\sum_{N=n, p} C_{T}^{N} \boldsymbol{\sigma}_{N}\right\rangle(\langle\ldots\rangle$ denoting the expectation value over a nuclear state with spin I), $\mu_{A}$ denotes the magnetic moment of the atom's nucleus (in units of the nuclear magneton), and the coefficients $C_{T}^{N}$ parametrize the tensorial P,T-odd electronnucleon interaction,

$$
\mathcal{H}_{T}=\frac{i G_{F}}{\sqrt{2}} \sum_{N=n, p} C_{T}^{N}\left(\bar{N} \sigma_{\mu \nu} \gamma_{5} N\right)\left(\bar{e} \sigma_{\mu \nu} e\right) .
$$

To further facilitate the comparison with other works, we note that the coefficient of the tensor interaction is typically parametrized via $\mathbf{d}_{A}=10^{-20} C_{C_{T}}\langle\boldsymbol{\sigma}\rangle C_{T} e \mathrm{~cm}$, implying

$$
\alpha_{C_{T}}=10^{-20} C_{C_{T}} \frac{\langle\boldsymbol{\sigma}\rangle \cdot \mathbf{I}}{I} e \mathrm{~cm} .
$$

The comparison is shown in table 2. We note that effects of interelectron correlations reduce $C_{C_{T}}$ by about a factor of $1 / 2$. Due to relations (3.3) and (3.5) these effects are 
expected to be qualitatively similar for the coefficient $\alpha_{C_{S}}$. In our result from the direct calculation electron correlation effects among the outermost 20 electrons of the $\mathrm{Hg}$ atom have been taken into consideration. There are two main sources for a potential difference between our value and the Coupled Cluster (CC) results via the phenomenological relation: 1) Our correlation model differs from the correlation models used in the CC calculations. 2) The phenomenological relation employs a uniform nuclear charge density whereas in our calculations a more realistic Gaussian charge distribution is used (see table 1) [28]. Since correlation effects tend to reduce the absolute value of $\alpha_{C_{S}}$ and our value is already about $15 \%$ below the CC results, it is reasonable to assume that no major correlation effects have been missed in our final computational model. The present difference is furthermore within the expected precision of this relation.

\section{Phenomenological consequences}

In order to explore the phenomenological consequences of our results, we follow two different strategies: (i) The common method to limit the corresponding Wilson coefficients assuming the absence of cancellations, i.e. setting all other contributions to zero. (ii) Limiting both $C_{S}$ and the electron EDM $d_{e}$ model-independently, i.e. allowing for cancellations between the two. This is achieved by combining information from the Mercury system with that of paramagnetic ones, following ref. [16], using the experimental results in table 3 . The key point in this strategy is that Mercury constrains a linear combination of $d_{e}$ and $C_{S}$ that is approximately orthogonal to the one constrained from paramagnetic systems, specifically ThO. This observation can be used to constrain $C_{S}$ and $d_{e}$, following a three-step argument:

1. The EDMs of paramagnetic systems are to good approximation dominated by contributions from $d_{e}$ and $C_{S}[45-47] .{ }^{1}$ While $C_{S}$ depends in general on the system under consideration, the combination that enters heavy atoms and molecules is to good approximation universal [16]. $C_{S}$ cannot be neglected model-independently: while NP models exist where the electron EDM clearly gives the leading contribution, this is not true in general. In Two-Higgs-Doublet models (2HDMs) for instance, the dominating Barr-Zee diagram for the electron EDM avoids a second small mass factor in addition to $m_{e}$, but as a two-loop diagram competes with a tree contribution to the S-PS-ne coupling that is suppressed by a light-quark mass and contains additional small factors like gauge couplings [13]. Schematically, we have $m_{u, d, s} \times$ tree vs. $m_{t} \times$ two-loop $\sim m_{t} /\left(16 \pi^{2}\right)^{2}$. Also in R-parity-violating SUSY models cancellations can occur, see for instance ref. [48].

2. Both contributions can in principle easily be taken into account, once two experiments with comparable sensitivity are available. The problem is that most of the constraints

\footnotetext{
${ }^{1}$ Strictly speaking also contributions from the Schiff moment and in some cases the magnetic quadrupole moment of the nucleus in paramagnetic systems could cancel these enhanced contributions. Given the large enhancement of the latter by $Z^{3} \sim 10^{5}$, this would however imply huge contributions in other systems, which are at least as severely constrained. However, formally a chain of cancellations in all constrained systems remains a possibility, due to the large number of potential sources.
} 


\begin{tabular}{|ccc|}
\hline Molecule & $\omega_{\exp } /(\mathrm{mrad} / \mathrm{s})$ & Refs. \\
\hline $\mathrm{HfF}^{+}$ & $0.3 \pm 2.7 \pm 0.6^{\mathrm{a}}$ & {$[39]$} \\
$\mathrm{ThO}$ & $2.6 \pm 4.8 \pm 3.2$ & {$[40,41]$} \\
$\mathrm{YbF}$ & $5.3 \pm 12.6 \pm 3.8$ & {$[42,43]$} \\
\hline $\mathrm{Atom}$ & $d_{A} /(e \mathrm{~cm})$ & Refs. \\
\hline $\mathrm{Tl}$ & $-(4.0 \pm 4.3) \times 10^{-25}$ & {$[44]$} \\
$\mathrm{Hg}$ & $(2.20 \pm 2.75 \pm 1.48) \times 10^{-30}$ & {$[14]$} \\
\hline
\end{tabular}

a Adapted to match the conventions used here.

Table 3. Experimental limits for the systems entering the global fit.

from paramagnetic systems are essentially parallel, so that typically fine-tuned solutions exist, where electron EDM and S-PS-ne contributions both oversaturate the experimental limit, but cancel to large extent in the measured observables. This leads to a situation where the model-independent approach yields a limit on the electron EDM that is about a factor of 10 weaker than the naive limit obtained when setting the S-PS-ne coupling to zero. This situation can be resolved by measurements on systems with different slopes, for example with relatively light atoms like $\mathrm{Rb}$ and very heavy ones like Fr. The recent measurement [39] already improves the situation significantly, as shown below.

3. In diamagnetic systems, there are several contributions to a potential EDM; assuming the presence of only electron EDM and S-PS-ne contributions here is clearly not a good description of, e.g., the Mercury EDM. However, the different hierarchy in this case can be used to turn the argument around: in diamagnetic systems both contributions are not enhanced, but strongly suppressed, because they yield a non-vanishing contribution only in combination with the hyperfine splitting. The sensitivity of Mercury to the electron EDM is about $3 \times 10^{8}$ weaker than in ThO. The relative sensitivity to other contributions, specifically the Schiff moment, to which for instance the quark (C)EDMs and the theta term contribute, and even tensor electron-nucleon couplings is much higher. This is why it is conservative to assume that these - often neglected - contributions saturate the experimental limit.

The conditions that have to be met for the resulting limit to be invalid are consequently very specific:

- The individual electron EDM and S-PS-ne contributions to the relevant paramagnetic systems would have to be larger than the experimental limits, but cancel in all of them sufficiently well.

- The electron EDM and S-PS-ne contributions to Hg would also have to be larger than the experimental limit, despite the massively different sensitivity.

- Since in the latter case a cancellation between the two contributions in $\mathrm{Hg}$ is not possible simultaneously with the paramagnetic systems, other contributions, that are 
each individually expected to be much larger than those from the electron EDM or S-PS-ne couplings, would have to combine in such a way that the net effect on the $\mathrm{Hg}$ EDM is again smaller than the experimental limit.

It is not impossible that all these things happen simultaneously, but since several cancellations on very different levels and in very different systems are necessary, we consider the limit resulting from our procedure conservative. Assumptions are made only on a subleading level, while in the literature it is very common to make them at the leading level, i.e. simply neglecting the S-PS-ne coupling. For convenience we provide below also the results without this assumption, i.e. when using the data from paramagnetic systems, only.

Note that the calculation presented here will remain useful even if the procedure outlined above should become unnecessary because of measurements in paramagnetic systems providing sufficiently precise and non-parallel constraints. Ultimately the goal should be a global analysis separating as many sources for EDMs as possible, see ref. [38] for a first attempt. Should both $d_{e}$ and $C_{S}$ be determined/limited from paramagnetic systems alone, the impact of the Mercury measurement on the remaining sources will increase, given a sufficiently precise determination of the corresponding coefficients.

Starting with strategy (i), i.e. assuming $C_{S}$ to give the only contribution to the Mercury EDM, we obtain from ref. [14] and eq. (3.2)

$$
C_{S}=-\left(0.8_{-1.2}^{+1.5}\right) \times 10^{-8}, \quad \text { or } \quad\left|C_{S}\right| \leq 3.2 \times 10^{-8}(95 \% \mathrm{CL}) \quad\left(C_{S} \text { only }\right) .
$$

This value is significantly larger than the one given in ref. [14], for two reasons: Heckel et al. used an indirectly obtained value for $\alpha_{C_{S}}$ [32], where moreover electron correlation effects have largely been neglected, which is much larger than our result on the absolute (and also larger than newer indirectly obtained results), and presumably used only the central value of that result. It is also significantly larger than the values obtained from ThO $\left(\left|C_{S}\right| \leq 0.7 \times 10^{-8}(95 \% \mathrm{CL})\right)$ and $\mathrm{HfF}^{+}\left(\left|C_{S}\right| \leq 1.8 \times 10^{-8}(95 \% \mathrm{CL})\right)$; however, as we will see below, the $\mathrm{Hg}$ result nevertheless improves the global fit significantly.

We perform global fits to the available data in table 3, using the theoretical inputs given in table 4 . The molecular measurements are typically expressed in terms of the angular frequency $\omega_{M}$, which can for our purposes be written as

$$
\begin{aligned}
\omega_{M} & =\left(-1.52 \operatorname{sgn}(\Omega) \frac{E_{\mathrm{eff}}}{\mathrm{GV} / \mathrm{cm}} \frac{d_{e}}{10^{-27} e \mathrm{~cm}}+2 \pi 10^{6} \Omega \frac{A_{M}}{Z_{M}} \frac{W_{S}}{\mathrm{kHz}} C_{S}\right)\langle\hat{n} \cdot \hat{z}\rangle \frac{\mathrm{mrad}}{\mathrm{s}} \\
& \equiv \alpha_{d_{e}}^{M} d_{e}+\alpha_{C_{S}}^{M} C_{S},
\end{aligned}
$$

where $E_{\text {eff }}$ the effective electric field, $\Omega=\left\langle\mathbf{J}_{e} \cdot \mathbf{n}\right\rangle$ is the projection of the total electronic angular momentum $\mathbf{J}_{e}$ on the molecule-fixed internuclear axis $\mathbf{n}, \hat{z}$ is the laboratory-frame $z$ axis defined by the direction of the external electric field, $A_{M}$ and $Z_{M}$ are the nucleon and the proton number of the heavy nucleus in the molecule $M$, respectively. The fit results are visualized in figure 1 . The shape of the individual constraints is determined by two factors: for a given ratio of the coefficients of $d_{e}$ and $C_{S}$, the slope of the twodimensional constraint is fixed and the experimental uncertainty determines the width of the associated band. Theoretical uncertainties add to that width, but mostly allow for 


\begin{tabular}{|cccccccc|}
\hline \multirow{2}{*}{ Molecule } & $\frac{E_{\text {eff }}}{(\mathrm{GV} / \mathrm{cm})}$ & ${\frac{W_{S}}{\mathrm{kHz}}}^{\mathrm{a}}$ & $\Omega$ & $\langle\hat{n} \cdot \hat{z}\rangle$ & $\frac{\alpha_{d_{e}}^{M}}{\mathrm{mrad} / \mathrm{s} /\left(10^{-27} \mathrm{ecm}\right)}$ & $\frac{\alpha_{C_{S}}^{M}}{10^{7} \mathrm{mrad} / \mathrm{s}}$ & Refs. \\
\hline $\mathrm{HfF}^{+}$ & $-23.0(0.9)$ & $20.4(0.8)$ & 1 & 1 & $34.9 \pm 1.4$ & $32.0 \pm 1.3$ & {$[39,49,50]$} \\
$\mathrm{ThO}$ & $-79.4(3.2)$ & $112.1(4.5)$ & 1 & 1 & $120.6 \pm 4.9$ & $181.6 \pm 7.3$ & {$[29,40,41,51]$} \\
$\mathrm{YbF}$ & $23.1(1.8)$ & $-40.5(3.2)$ & $1 / 2$ & 0.558 & $-19.6 \pm 1.5$ & $-17.6 \pm 2.0$ & {$[42,43,52,53]$} \\
\hline \multirow{2}{*}{$\mathrm{Atom}$} & & $\alpha_{d_{e}}^{A}$ & & $\frac{\alpha_{C_{S}}^{A}}{10^{-20} e \mathrm{~cm}}$ & Refs. \\
\hline $\mathrm{Fr}$ & $885 \pm 35$ & $1090 \pm 17$ & {$[54-56]$} \\
$\mathrm{Tl}$ & $-573 \pm 20^{\mathrm{b}}$ & $-700 \pm 35^{\mathrm{c}}$ & {$[60,61]$} \\
$\mathrm{Cs}$ & $120 \pm 3$ & & $78 \pm 2$ & {$[54,59,61,62]$} \\
$\mathrm{Rb}$ & & $25.7 \pm 0.8$ & & $11.0 \pm 0.2$ & {$[54,62]$} \\
\hline $\mathrm{Hg}$ & $0.012 \pm 0.012$ & $-0.028 \pm 0.006$ & {$[63]$, this work } \\
\hline
\end{tabular}

${ }^{\text {a }}$ Note the existence of different conventions in the literature; for instance, the coefficient $W_{S}$ used here is called $W_{T, P}$ in ref. [49], while $W_{S}$ in that reference denotes the product $A / Z W_{T, P}$ appearing in eq. (4.2).

${ }^{\mathrm{b}}$ For discussions regarding this value, see also refs. [57, 58]. Note that the global fit is not affected by this discussion.

${ }^{c}$ See also ref. [59].

Table 4. Relevant information regarding the systems under consideration. $\alpha_{d_{e}, C_{S}}^{A}$ are defined in analogy to eq. (4.3) as $d_{A}=\alpha_{d_{e}} d_{e}+\alpha_{C_{S}} C_{S}$.

a range of slopes, which yields fan-shaped constraints. Hence, the more important the theoretical uncertainties, the more fan-shaped the constraint will be, the most obvious example being $\mathrm{Hg}$. Apart from the individual constraints from the paramagnetic systems $\mathrm{ThO}, \mathrm{HfF}^{+}, \mathrm{YbF}, \mathrm{Tl}$, we show the one from $\mathrm{Hg}$, as well as the combinations of only the paramagnetic constraints and the global fit to all systems. The fit to only paramagnetic systems is massively improved by the $\mathrm{HfF}^{+}$measurement: before this measurement it extended essentially over the whole green area. Our result for Mercury, including only the contributions from $d_{e}$ and $C_{S}$ as discussed above, is seen to additionally improve the fit, reducing the model-independent limits for both quantities significantly. This is due to the constraint being essentially orthogonal to those from the paramagnetic systems: we obtain for the paramagnetic systems a range $\alpha_{C_{S}}^{M, A} / \alpha_{d_{e}}^{M, A} \in[0.4,1.5] \times 10^{-20} e \mathrm{~cm}$, while for Mercury we obtain conservatively $\alpha_{C_{S}}^{\mathrm{Hg}} / \alpha_{d_{e}}^{\mathrm{Hg}}<-0.9 \times 10^{-20} e \mathrm{~cm}$. The latter ratio will be more precisely determined once the coefficient for the electron EDM in $\mathrm{Hg}$ is known better, which is work in progress; here we assumed an uncertainty of $100 \%$, given the unreliable estimate. This will also improve the determination of $d_{e}$ and $C_{S}$. In table 5 we give the numerical results of both fits (global and paramagnetic only), including the effective correlations between the results for $d_{e}$ and $C_{S}$, as well as the corresponding upper limits. While the individual constraints from $\mathrm{Hg}$ are weaker than those extracted from $\mathrm{ThO}$ and $\mathrm{HfF}^{+}$, its inclusion in the global fit results in model-independent limits about a factor of two stronger than those from the paramagnetic systems alone. 


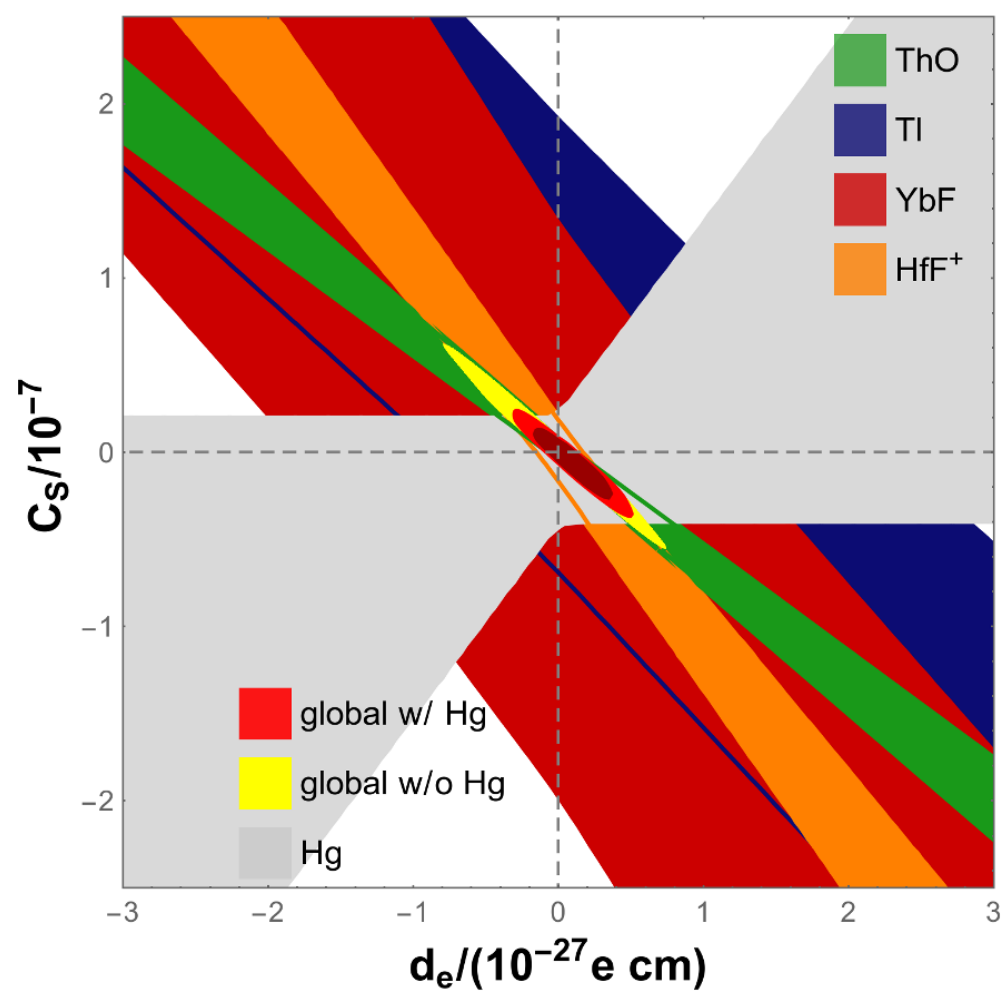

Figure 1. Fit to the available data from paramagnetic systems plus the constraint from Mercury, using the result presented in this work. The bands from the individual constraints as well as the global fit without Mercury correspond to $95 \%$ CL, the global fit with Mercury to $68 \%$ and $95 \%$ CL. These bands include both experimental and theoretical uncertainties. Individual constraints have 1 effective degree of freedom, the global fits 2 .

\begin{tabular}{|cccc|}
\hline Fit & $d_{e} / 10^{-28} e \mathrm{~cm}$ & $C_{S} / 10^{-8}$ & Correlation \\
\hline \multirow{2}{*}{ global (w/ Hg) } & $1.1 \pm 1.7$ & $-0.6 \pm 1.2$ & $-96 \%$ \\
& $\left|d_{e}\right| \leq 3.8$ & $\left|C_{S}\right| \leq 2.7$ & \\
\hline \multirow{2}{*}{ param. only (w/o Hg) } & $-0.9 \pm 3.2$ & $0.8 \pm 2.4$ & $-99 \%$ \\
& $\left|d_{e}\right| \leq 6.4$ & $\left|C_{S}\right| \leq 4.9$ & \\
\hline
\end{tabular}

Table 5. Fit results for the global fit, using our result for $\mathrm{Hg}$, and the fit using only the results from paramagnetic systems. The former yields limits about a factor of two stronger than the latter.

\section{Conclusions and outlook}

We performed global fits to the available data constraining the electron EDM and the S-PS-ne nucleon-electron coupling entering heavy atoms and molecules, using up-to-date calculations of the atomic and molecular structures. The inclusion of the recent result on $\mathrm{HfF}^{+}$ions improves drastically the fit to paramagnetic systems, only. As pointed out in ref. [16], diamagnetic systems can be used to improve this fit additionally; while the corresponding contributions are heavily suppressed in this case, diamagnetic systems have the advantage of constraining in some cases combinations orthogonal to those accessible in 


\begin{tabular}{|ccc|}
\hline Atom & \multicolumn{2}{c|}{ Limits for $\left|d_{A}\right| / 10^{-26} e \mathrm{~cm}$} \\
& Inferred (this work) & Experimental \\
\hline $\mathrm{Rb}$ & $0.7(1.2)$ & $10^{8}(1200)[64,65]$ \\
$\mathrm{Cs}$ & $2.7(4.2)$ & $1400[66]$ \\
$\mathrm{Fr}$ & $13.0(14.8)$ & - \\
\hline Molecule & Limits for & $\left|\omega_{M}\right| /(\mathrm{mrad} / \mathrm{s})^{*}$ \\
\hline $\mathrm{YbF}$ & $3.7(5.6)$ & $27.8[42,43,67]$ \\
\hline
\end{tabular}

Table 6. Model-independent limits for paramagnetic systems from our global fits; the numbers in brackets correspond to the fit including paramagnetic systems, only.

paramagnetic systems. As an illustration we performed the first direct calculation of the coefficient of the S-PS-ne coupling in Mercury, including the effect of electron correlations. In combination with the recently improved experimental limit for this system we obtain limits on both the electron EDM and the S-PS-ne coupling of about a factor of two stronger than from paramagnetic systems alone, see table 5 .

Having a model-independent determination of both quantities determining the EDMs of paramagnetic systems in hand, we proceed to evaluate the impact on on-going searches. The global fits imply non-trivial upper limits for every paramagnetic system that is not effectively constraining the fits in figure 1 . These limits, given in table 6 , indicate the necessary precision for a given system to contribute significantly to the global fit or the fit to paramagnetic systems, only (given in parentheses). A significant result above both limits would indicate an experimental problem, unless the dominance of the enhanced contributions in paramagnetic systems or the calculations of their coefficients are invalidated by some as-of-yet unknown mechanism. A measurement below the limit from the fit to paramagnetic systems, but above the one from the global fit, could in principle also indicate the contrived situation with a series of cancellations, described at the beginning of section 4 .

In the future, it is to be expected that measurements in paramagnetic systems alone will yield sufficiently precise results to limit or determine the two contributions discussed here by themselves. In that case our calculations will serve to improve the model-independent determination of hadronic contributions to diamagnetic EDMs in the context of a global fit extending over the whole set of $\mathrm{P}, \mathrm{T}$-odd interactions.

\section{Acknowledgments}

This research was supported by the DFG cluster of excellence "Origin and Structure of the Universe". The authors are grateful to the Mainz Institute for Theoretical Physics (mitp) for its hospitality and its partial support during the completion of this work. 
Open Access. This article is distributed under the terms of the Creative Commons Attribution License (CC-BY 4.0), which permits any use, distribution and reproduction in any medium, provided the original author(s) and source are credited.

\section{References}

[1] M.B. Gavela, P. Hernández, J. Orloff and O. Pene, Standard model CP-violation and baryon asymmetry, Mod. Phys. Lett. A 9 (1994) 795 [hep-ph/9312215] [INSPIRE].

[2] P. Huet and E. Sather, Electroweak baryogenesis and standard model CP-violation, Phys. Rev. D 51 (1995) 379 [hep-ph/9404302] [INSPIRE].

[3] M.B. Gavela, P. Hernández, J. Orloff, O. Pene and C. Quimbay, Standard model CP-violation and baryon asymmetry. Part 2: Finite temperature, Nucl. Phys. B 430 (1994) 382 [hep-ph/9406289] [INSPIRE].

[4] J.S.M. Ginges and V.V. Flambaum, Violations of fundamental symmetries in atoms and tests of unification theories of elementary particles, Phys. Rept. 397 (2004) 63 [physics/0309054] [INSPIRE].

[5] M. Pospelov and A. Ritz, Electric dipole moments as probes of new physics, Annals Phys. 318 (2005) 119 [hep-ph/0504231] [INSPIRE].

[6] M. Raidal et al., Flavour physics of leptons and dipole moments, Eur. Phys. J. C 57 (2008) 13 [arXiv: 0801.1826] [INSPIRE].

[7] T. Fukuyama, Searching for New Physics beyond the Standard Model in Electric Dipole Moment, Int. J. Mod. Phys. A 27 (2012) 1230015 [arXiv:1201.4252] [INSPIRE].

[8] J. de Vries, E. Mereghetti, R.G.E. Timmermans and U. van Kolck, The Effective Chiral Lagrangian From Dimension-Six Parity and Time-Reversal Violation, Annals Phys. 338 (2013) 50 [arXiv: 1212.0990] [InSPIRE].

[9] J. Engel, M.J. Ramsey-Musolf and U. van Kolck, Electric Dipole Moments of Nucleons, Nuclei and Atoms: The Standard Model and Beyond, Prog. Part. Nucl. Phys. 71 (2013) 21 [arXiv: 1303.2371] [INSPIRE].

[10] J. Bsaisou, U.-G. Meißner, A. Nogga and A. Wirzba, P- and T-Violating Lagrangians in Chiral Effective Field Theory and Nuclear Electric Dipole Moments, Annals Phys. 359 (2015) 317 [arXiv:1412.5471] [INSPIRE].

[11] T. Chupp, P. Fierlinger, M. Ramsey-Musolf and J. Singh, Electric Dipole Moments of the Atoms, Molecules, Nuclei and Particles, arXiv:1710.02504 [INSPIRE].

[12] N. Yamanaka, B.K. Sahoo, N. Yoshinaga, T. Sato, K. Asahi and B.P. Das, Probing exotic phenomena at the interface of nuclear and particle physics with the electric dipole moments of diamagnetic atoms: A unique window to hadronic and semi-leptonic CP-violation, Eur. Phys. J. A 53 (2017) 54 [arXiv:1703.01570] [InSPIRE].

[13] M. Jung and A. Pich, Electric Dipole Moments in Two-Higgs-Doublet Models, JHEP 04 (2014) 076 [arXiv: 1308.6283] [INSPIRE].

[14] B. Graner, Y. Chen, E.G. Lindahl and B.R. Heckel, Reduced Limit on the Permanent Electric Dipole Moment of Hg199, Phys. Rev. Lett. 116 (2016) 161601 [Erratum ibid. 119 (2017) 119901] [arXiv: 1601.04339] [INSPIRE]. 
[15] V.A. Dzuba, V.V. Flambaum and C. Harabati, Relations between matrix elements of different weak interactions and interpretation of the parity-nonconserving and electron electric-dipole-moment measurements in atoms and molecules, Phys. Rev. A 84 (2011) 052108 [Erratum ibid. 85 (2012) 029901] [arXiv:1109.6082].

[16] M. Jung, A robust limit for the electric dipole moment of the electron, JHEP 05 (2013) 168 [arXiv: 1301.1681] [INSPIRE].

[17] L.I. Schiff, Measurability of Nuclear Electric Dipole Moments, Phys. Rev. 132 (1963) 2194 [INSPIRE].

[18] I.B. Khriplovich and S.K. Lamoreaux, CP Violation Without Strangeness, Springer, (1997).

[19] S. Knecht, H.J. Aa. Jensen and T. Fleig, Large-Scale Parallel Configuration Interaction. II. Two- and four-component double-group general active space implementation with application to BiH, J. Chem. Phys. 132 (2010) 014108.

[20] V.V. Flambaum and I.B. Khriplovich, New Limits on the Electron Dipole Moment and T Nonconserving Electro-Nucleon Interaction, Sov. Phys. JETP 62 (1985) 872 [INSPIRE].

[21] T. Fleig and M.K. Nayak, Electron Electric Dipole Moment and Hyperfine Interaction Constants for ThO, J. Molec. Spectrosc. 300 (2014) 16 [arXiv:1401.2284] [INSPIRE].

[22] M. Denis et al., Theoretical study on $T h F^{+}$, a prospective system in search of time-reversal violation, New J. Phys. 17 (2015) 043005.

[23] V.A. Dzuba, V.V. Flambaum and S.G. Porsev, Calculations of the $(P, T)$-odd electric dipole moments for the diamagnetic atoms ${ }^{129} \mathrm{Xe},{ }^{171} \mathrm{Yb},{ }^{199} \mathrm{Hg},{ }^{211} \mathrm{Rn}$, and ${ }^{225} \mathrm{Ra}$, Phys. Rev. A 80 (2009) 032120 [arXiv: 0906.5437] [INSPIRE].

[24] N.J. Stone, Table of nuclear magnetic dipole and electric quadrupole moments, IAEA Nuclear Data Section Vienna International Centre, Vienna, Austria, (2014), INDC International Nuclear Data Committee.

[25] K.G. Dyall, Relativistic double-zeta, triple-zeta, and quadruple-zeta basis sets for the $5 d$ elements Hf-Hg, Theoret. Chim. Acta 112 (2004) 403.

[26] K.G. Dyall and A.S.P. Gomes, Revised relativistic basis sets for the $5 d$ elements $H f-H g$, Theoret. Chim. Acta 125 (2010) 97.

[27] A. Kramida, Yu. Ralchenko, J. Reader and and NIST ASD Team, NIST Atomic Spectra Database (ver. 5.3), http://physics.nist.gov/asd, (2017, February 21), National Institute of Standards and Technology, Gaithersburg, MD., U.S.A., (2015).

[28] L. Visscher and K.G. Dyall, Dirac-Fock Atomic Electronic Structure Calculations using Different Nuclear Charge Distributions, Atom. Data Nucl. Data Tabl. 67 (1997) 207.

[29] M. Denis and T. Fleig, In search of discrete symmetry violations beyond the standard model: Thorium monoxide reloaded, J. Chem. Phys. 145 (2016) 214307.

[30] T. Fleig, M.K. Nayak and M.G. Kozlov, TaN, a molecular system for probing $\mathcal{P}, \mathcal{T}$-violating hadron physics, Phys. Rev. A 93 (2016) 012505 [arXiv: 1512.08729] [INSPIRE].

[31] M.G. Kozlov, New Limit on the Scalar P, T Odd Electron Nucleus Interaction, Phys. Lett. A 130 (1988) 426 [INSPIRE].

[32] A.-M. Martensson-Pendrill, Calculation of a P-and T-Nonconserving Weak Interaction in Xe and Hg with Many-Body Perturbation Theory, Phys. Rev. Lett. 54 (1985) 1153 [InSPIRE]. 
[33] L. Radžiūtè, G. Gaigalas, P. Jönsson and J. Bieroń, Electric dipole moments of superheavy elements - A case study on copernicium, Phys. Rev. A 93 (2016) 062508 [arXiv: 1508.03974] [INSPIRE].

[34] K.V.P. Latha, D. Angom, B.P. Das and D. Mukherjee, Probing CP-violation with the electric dipole moment of atomic mercury, Phys. Rev. Lett. 103 (2009) 083001 [arXiv:0902.4790] [INSPIRE].

[35] Y. Singh and B.K. Sahoo, Rigorous limits for hadronic and semi-leptonic CP-violating coupling constants from the electric dipole moment of ${ }^{199} \mathrm{Hg}$, Phys. Rev. A 91 (2015) 030501 [arXiv: 1408.4337] [INSPIRE].

[36] B. Sahoo, Improved limits on the hadronic and semihadronic CP violating parameters and role of a dark force carrier in the electric dipole moment of ${ }^{199} \mathrm{Hg}$, Phys. Rev. D 95 (2017) 013002 [arXiv: 1612.09371] [INSPIRE].

[37] B.K. Sahoo and B.P. Das, Relativistic Normal Coupled-Cluster Theory for Accurate Determination of Electric Dipole Moments of Atoms: First application to ${ }^{199} \mathrm{Hg}$ atom, Phys. Rev. Lett. 120 (2018) 203001 [arXiv:1801.07045].

[38] T. Chupp and M. Ramsey-Musolf, Electric Dipole Moments: A Global Analysis, Phys. Rev. C 91 (2015) 035502 [arXiv:1407.1064] [INSPIRE].

[39] W.B. Cairncross et al., Precision Measurement of the Electron's Electric Dipole Moment Using Trapped Molecular Ions, Phys. Rev. Lett. 119 (2017) 153001 [arXiv:1704.07928] [INSPIRE].

[40] ACME collaboration, J. Baron et al., Order of Magnitude Smaller Limit on the Electric Dipole Moment of the Electron, Science 343 (2014) 269 [arXiv:1310.7534] [INSPIRE].

[41] J. Baron et al., Methods, Analysis, and the Treatment of Systematic Errors for the Electron Electric Dipole Moment Search in Thorium Monoxide, New J. Phys. 19 (2017) 073029.

[42] J.J. Hudson, D.M. Kara, I.J. Smallman, B.E. Sauer, M.R. Tarbutt and E.A. Hinds, Improved measurement of the shape of the electron, Nature 473 (2011) 493 [INSPIRE].

[43] D.M. Kara, I.J. Smallman, J.J. Hudson, B.E. Sauer, M.R. Tarbutt and E.A. Hinds, Measurement of the electron's electric dipole moment using YbF molecules: methods and data analysis, New J. Phys. 14 (2012) 103051 [arXiv:1208.4507] [INSPIRE].

[44] B.C. Regan, E.D. Commins, C.J. Schmidt and D. DeMille, New limit on the electron electric dipole moment, Phys. Rev. Lett. 88 (2002) 071805 [INSPIRE].

[45] P.G.H. Sandars, The electric dipole moment of an atom, Phys. Lett. 14 (1965) 194.

[46] P.G.H. Sandars, Enhancement factor for the electric dipole moment of the valence electron in an alkali atom, Phys. Lett. 22 (1966) 290.

[47] V.V. Flambaum, On enhancement of the electron electric dipole moment in heavy atoms, Yad. Fiz. 24 (1976) 383 [inSPIRE].

[48] N. Yamanaka, T. Sato and T. Kubota, Linear programming analysis of the R-parity violation within EDM-constraints, JHEP 12 (2014) 110 [arXiv:1406.3713] [INSPIRE].

[49] L.V. Skripnikov, Communication: Theoretical study of $\mathrm{HfF}^{+}$cation to search for the T,P-odd interactions, J. Chem. Phys. 147 (2017) 021101.

[50] T. Fleig, $\mathcal{P}, \mathcal{T}$-odd and magnetic hyperfine-interaction constants and excited-state lifetime for $H_{f F}^{+}$, Phys. Rev. A 96 (2017) 040502 [arXiv: 1706.02893] [INSPIRE]. 
[51] L.V. Skripnikov, Combined 4-component and relativistic pseudopotential study of ThO for the electron electric dipole moment search, J. Chem. Phys. 145 (2016) 214301.

[52] M. Abe, G. Gopakumar, M. Hada, B.P. Das, H. Tatewaki and D. Mukherjee, Application of relativistic coupled-cluster theory to the effective electric field in YbF, Phys. Rev. A 90 (2014) 022501.

[53] A. Sunaga, M. Abe, M. Hada and B.P. Das. Relativistic coupled-cluster calculation of the electron-nucleus scalar-pseudoscalar interaction constant $W_{S}$ in YbF, Phys. Rev. A 93 (2016) 042507.

[54] B.M. Roberts, V.A. Dzuba and V.V. Flambaum, Double-core-polarization contribution to atomic parity-nonconservation and electric-dipole-moment calculations, Phys. Rev. A 88 (2013) 042507 [arXiv:1309.3371] [INSPIRE].

[55] D. Mukherjee, B.K. Sahoo, H.S. Nataraj and B.P. Das. Relativistic coupled cluster (rcc) computation of the electric dipole moment enhancement factor of francium due to the violation of time reversal symmetry, J. Phys. Chem. A 113 (2009) 12549.

[56] L.V. Skripnikov, D.E. Maison and N.S. Mosyagin, Scalar-pseudoscalar interaction in the francium atom, Phys. Rev. A 95 (2017) 022507 [arXiv:1611.09103] [INSPIRE].

[57] H.S. Nataraj, B.K. Sahoo, B.P. Das and D. Mukherjee, A Reappraisal of the Electric Dipole Moment Enhancement Factor for Thallium, Phys. Rev. Lett. 106 (2011) 200403 [arXiv: 1005.1797] [INSPIRE].

[58] H.S. Nataraj, B.K. Sahoo, B.P. Das and D. Mukherjee, Brief remarks on "Electric dipole moment enhancement factor of thallium", arXiv:1202.5402.

[59] B K Sahoo, B P Das, R K Chaudhuri, D Mukherjee and E P Venugopal, Atomic electric-dipole moments from Higgs-boson-mediated interactions, Phys. Rev. A 78 (2008) 10501.

[60] S.G. Porsev, M.S. Safronova and M.G. Kozlov, Electric dipole moment enhancement factor of thallium, Phys. Rev. Lett. 108 (2012) 173001 [arXiv:1201.5615] [INSPIRE].

[61] V.A. Dzuba and V.V. Flambaum, Calculation of the (T,P)-odd Electric Dipole Moment of Thallium, Phys. Rev. A 80 (2009) 062509 [arXiv:0909.0308] [InSPIRE].

[62] H.S. Nataraj, B.K. Sahoo, B.P. Das and D. Mukherjee, Intrinsic Electric Dipole Moments of Paramagnetic Atoms: Rubidium and Cesium, Phys. Rev. Lett. 101 (2008) 033002.

[63] A.M. Mårtensson-Pendrill and P. Öster, Calculations of Atomic Electric Dipole Moments, Phys. Scripta 36 (1987) 444.

[64] E.S. Ensberg. Experimental upper limit for the permanent electric dipole moment of $R b^{85}$ by optical-pumping techniques, Phys. Rev. 153 (1967) 36.

[65] F.R. Huang-Hellinger Jr., A Search for a Permanent Electric Dipole Moment in Rubidium, Ph.D. Thesis, University of Washington, Seattle, U.S.A. (1987).

[66] S.A. Murthy, D. Krause, Z.L. Li and L.R. Hunter, New Limits on the Electron Electric Dipole Moment from Cesium, Phys. Rev. Lett. 63 (1989) 965 [INSPIRE].

[67] J.J. Hudson, B.E. Sauer, M.R. Tarbutt and E.A. Hinds, Measurement of the electron electric dipole moment using YbF molecules, Phys. Rev. Lett. 89 (2002) 023003 [hep-ex/0202014] [INSPIRE]. 